am selben Tage auf einer Wiese Gymnadenia conopsea v. albiflora. Stengel war $10-15 \mathrm{~cm}$. hoch, lichtgrün, die Blüthen reinweiss, Sporn durchsichtig, die Seitenzipfel des Labellums schmal-lineal, die Deckblätter grün. - Bellis annua L. fand ich bei Buccari auf Grasplätzen noch im Jahre 1878. Ein sehr interessanter Fund ist Mentha seriata Kerner, welche ich bei Buccarizza am 29. Juli entdeckte, und welche mir v. Borbás an Ort und. Stelle 30. Juli determinirte.

Von Rosen, welche für die Flora croatica neu sind, fand. ich in der Umgebung von Buccari ausser Rosa Hirciana H. Br. folgende:

Rosa glauca f. Sandbergi Christ. Auf felsigen Abhängen bei St. Jacob, unweit von Portorè. Mai. Die typische Form dieser Rose fand v. Borbás bei Begovo Razdolje, unweit Mrkopalj am Plateau. (Primitiae monographiae Rosarum imperii Hungarici [1880] p. 459.)

$\boldsymbol{R}$. rubella Sm. Auf Felsen längs der Karolinenstrasse bei Zlobin und am Fusse de Tuhobić-Berges. Juni.

f. inermis H. Braun (in litt. 1882). Anf Bergwiesen oberhalb Drvenik in Vinodol mit $R$. spinosissima L. Juni.

R. rubiginosa L. f. Gremlii Christ. in "Die Rosen d. Schweiz" (1873) p. 107. - Diese Rosa entdeckte "ich auf steinigen Stellen bei Vitoševo und Kukuljanovo. Mai.

R. sepium Thuill. f. mentita (Déségl.). Auf einem felsigen Abhange bei Buccari. Juni.

R. dumetorum Thuill. f. solstitialis (Bess.). Bei Kuljanovo längs eines Steinwalles. Mai.

$\boldsymbol{R}$. dumalis Bechst. f. rotundata $\mathrm{H}$. Br. (in litt. 1882). In einem Weingarten am Fusse des Gipfels Ravna bei Buccari mit $\boldsymbol{R}$. canina L. f. pallens Déségl. Mai.

$\boldsymbol{R}$. canina L. f. Lutetiana Lém.-Willk. Führer in d. Reiche d. Pflanzen Dentschlands, Oesterreichs u. d. Schweiz (1882), p. 645. Auf dem Kalvarienberge bei Buccari. Mai.

Herrn Heinrich Braun in Wien, welcher freundlichst die Revision dieser und anderer Rosen aus Croatien übernahm, spreche ich nochmals meinen besten Dank aus.

Buccari, am 10. Angust 1883.

\title{
Flora von Meran im August a. c.
}

Von Prof. Dr. Entleutner.

Noch vor Tagesanbruch wandern wir über den Weiler Töll nach Partschins. Von dort aus führt uns ein ziemlich beschwerlicher Weg in das Zielthal hinein. Nach 1 Stunde haben wir den Wasserfall des Zielbaches erreicht, in dessen Nähe man Asplenium germa- 
nicum Weiss f: alpestris findet. War der Weg bisher schon steil und beschwerlich, so wird er es von jetzt an erst recht. Anfangs am linken, dann am rechten Ufer des Zielbaches klimmen wir empor bis zur dritten und obersten Zielalm.

Die alpine Vegetation, welche wir da antreffen, lohnt reichlich die Mühe des Aufstieges. Hier sehen wir nämlich blühende und zum Theil auch schon abgeblühte Exemplare von: Ranunculus glacialis et parnassifolius, Arabis caerulea Haenke, Cerastium latifolium, Stellaria cerastoides, Thesium alpinum, Hieracium porrifolium, $\boldsymbol{H}$. alpinum, Schraderi Schleich, glanduliferum Hoppe und albidum Vill., Achillea Clavenae et atrata, Anthemis alpina, Gnaphalium supinum et carpaticum Wahlb., Artemisia spicata Wulf., Erigeron uniflorus, Aronicum scorpioides Koch, Senecio Doronicum, Leontodon Tarasaci Lois. et pyrenaicum Gouan. Saxifraga caesia, bryoides, androsacea, muscoides Wulf. und exarata Vill., Androsace obtusifolia All., Gentiana bavarica, punctata, nivalis, imbricata Froel. und brachyphylla Vill., Phyteuma Sieberi Spreng. und pauciflorum, Geum reptans, Pedicularis rostrata, recutita und asplenifolia Floerke, Hedysarum obscumum, Potentilla grandiflora und salisburgensis Haenke, Gaya simplex Gaud., Athamanta cretensis, Epilobium alpinum, Rhinanthus alpinus Baumg., Chamaeorchis alpina Rich., Coeloglossum viride Hartm., Gagea Liottardi Schult., Lloydia serotina Salisb., Salix herbacea, arbuscula und retusa, Juncus Jacquini, triglumis, Hostii und alpinus Vill., Luzula lutea DC., Festuca pumila und Scheuchzeri Gaud., Sesleria disticha Pers., Koeleria hirsuta Gaud., Selaginella spinulosa Al. Br.

Auf den Spronseralpen blühen: Statice alpina Hoppe, Draba frigida Saut., Sempervivum Wulfenii Hoppe, Saxifraga sedoides, Laserpitium hirsutum Lam., Aronicum Clusii Koch, Poa laxa Haenke, Calamagrostis Halleriana DC. und tenella Host, Festuca pilosa Hall., Lycopodium alpinum.

Besteigen wir die Granitpyramide des Ifinger, so finden wir: Draba aizoides, Sedum repens, Cerastium alpinum, Senecio carniolicus Willd. und incanus, Cirsium ambiguum All., Circaèa alpina, Epilobium origanifolium, Androsace glacialis Hoppe, Euphrasia mirima Schleich., Poa minor Gaud. und alpina, Agrostis alpina Scop. und rupestris All., Festuca Halleri All.

Doch nun herab von den Bergen ins Meraner Thal. Hier sieht man: Nepeta Cataria, Alsine Jacquini Koch, Pulicaria vulgaris Gaertn., Lappa major Gaertn., Aster Amellus, Hieracium boreale Fries, Sedum reflexum, Circaea lutetiana, Dianthus monspessulanus, Chenopodium polyspermum, Stachys germanica, Cyperus longus, flavescens, fuscus und Monti, Eragrostis megastuchya Link., poaeoides Beauv. und pilosa Beauv. 